\title{
Alcohol induced neurocognitive impairment (wernicke - korsakoff): A hidden syndrome
}

\author{
Prabhoo Dayal ${ }^{1,}$, Ankur Sachdeva ${ }^{1}$, Mina Chandra ${ }^{2}$, Kishore Hindustani ${ }^{3}$, Kuljeet Singh Anand ${ }^{4}$ \\ ${ }^{1}$ National Drug Dependence Treatment Centre (WHO Collaborating centre), All India Institute of Medical Sciences (AIIMS), Ansari Nagar, \\ New Delhi-110029, India \\ ${ }^{2}$ Department of Psychiatry and Drug De-addiction, Post Graduate Institute of Medical Education \& and Research (PGIMER) and Dr Ram \\ Manohar Lohia Hospital, New Delhi-110001, India \\ ${ }^{3}$ Department of Psychiatry, VMMC and Safdarjang Hospital, New Delhi, India \\ ${ }^{4}$ Department of Neurology, Post Graduate Institute of Medical Education \& and Research (PGIMER) and Dr Ram Manohar Lohia Hospital, \\ New Delhi-110001, India
}

\section{Email address:}

drpd@rediffmail.com (P. Dayal)

\section{To cite this article:}

Prabhoo Dayal, Ankur Sachdeva, Mina Chandra, Kishore Hindustani, Kuljeet Singh Anand. Alcohol Induced Neurocognitive Impairment (Wernicke - Korsakoff): A Hidden Syndrome. Clinical Medicine Research. Special Issue: Recent Research in Dementia.

Vol. 4, No. 2-1, 2015, pp. 15-23. doi: 10.11648/j.cmr.s.2015040201.13

\begin{abstract}
Wernicke's encephalopathy (WE) is an acute neuro-psychiatric syndrome due to inadequate supply of thiamine (vitamin B1) to the brain which leads to significant morbidity and mortality. Although alcohol use is the most common predisposing factor but Wernicke's encephalopathy can occur in any patient with nutritional deficiency conditions such as hyperemesis gravidarum, hemodialysis, malignancy, use of total parenteral nutrition without adequate thiamine, and abdominal surgery. In a developing country, there are more chances of thiamine deficiency, because of poor intake of nutrients in routine diet due to economic reasons and local customs and cultural practices concerning the processing and cooking of rice and other foodstuffs. The national household survey of drug use in India (2004) found prevalence of alcohol use in about $21.4 \%$ of male population between 18-40 age group. Most of the emergency physicians and general practitioners are not well sensitized about neuropsychiatric disorders in patients with alcohol use disorders. Such patients are still under diagnosed. Till date, no such studies are available about treatment of wernicke-korsakoff syndrome in Indian population. Educating clinicians, specially emergency physicians about evaluation and treatment of Wernicke-Korsakoff syndrome is as important as to educate people to eat well balanced, mixed diet containing thiamine rich food as most of these patients receive emergency treatment but are frequently unrecognized. If undiagnosed or inadequately treated, it is likely to proceed to Korsakoff syndrome. Therefore best treatment for Wernicke-Korsakoff syndrome is prompt diagnosis and adequate treatment.
\end{abstract}

Keywords: Alcohol, Wernicke's encephalopathy, Thiamine (Vitamin B1), Korsakoff Syndrome, Neurocognitive Impairment, Treatment

\section{Introduction}

Wernicke's encephalopathy (WE) is an acute neuropsychiatric condition caused by an inadequate supply of thiamine (vitamin B1) to the brain. Carl Wernicke described this distinctive entity in 1881 as acute superior hemorrhagic polioencephalitis .It involves an acute Wernickeencephalopathy phase, followed by the development of a chronic Korsakoff's syndrome phase. Although WE and KS were discovered separately, these two syndromes are usually referred to under one name, Wernicke-Korsakoff syndrome, due to the fact that they are part of the same etiology and because the onset of KS usually follows WE if left untreated. The link to thiamine deficiency as a causative factor was discovered approximately 50 years later in the 1940 s by Campbell and Russell (1).

According to autopsy-based studies, the disorder is still greatly under diagnosed in adults. Although alcoholism is the most common predisposing factor but Wernicke's encephalopathy may occur in any patient with nutritional deficiency conditions such as hyperemesis gravidarum, hemodialysis, malignancy, use of total parenteral nutrition 
without adequate thiamine, and abdominal surgery (2-7). Thiamine deficiency can occur as a result of inadequate dietary intake, reduced absorption of thiamine, and a reduction in the rate of conversion to the active metabolite. One or more of these factors may be implicated. WE is reversible if treated with a timely and adequate dose of parenteral thiamine. If it is undiagnosed or inadequately treated, it is likely to proceed to the chronic state, Korsakoff's syndrome (KS) which is associated with significant morbidity and mortality. It follows from this that the best treatment for KS is timely recognition of WE, and appropriate intervention and prevention.

In this review, we will provide an update on the factors and clinical settings that predispose to Wernicke's encephalopathy, and discuss the most recent insights into epidemiology, pathophysiology, genetics, diagnosis, and treatment. We also update the optimum dose of parenteral thiamine required for prophylaxis and treatment of Wernicke's encephalopathy and prevention of Korsakoff syndrome associated with alcohol misuse.

\section{Epidemiology}

Information on the prevalence of Wernicke's encephalopathy has been reported mostly from retrospective autopsy studies of hospitalized patients (8-12). In adults, autopsy studies have reported a higher prevalence of Wernicke's encephalopathy lesions $(0 \cdot 8-2 \cdot 8 \%)$ than is predicted by clinical studies $(0 \cdot 04-0 \cdot 13 \%)(13,14,15)$ and that $12.5 \%$ of alcoholics have these brain lesions (16). It means, only a fraction of cases (approximately 15\%) are diagnosed ante-mortem $(9,17,18)$. Unfortunately, in alcoholics (13) Wernicke's encephalopathy confirmed at autopsy had been missed by routine clinical examination in $75-80 \%$ of cases. About $80 \%$ of patients with acute Wernicke's encephalopathy develop Korsakoff syndrome (19). Incidence and prevalence of Korsakoff syndrome would seemingly mimic that of acute Wernicke's encephalopathy. Wernicke's encephalopathy is more common in males than in females (male-to-female ratio 1.7 to 1) (20). The estimated mortality is $17 \%$ (21). Asians tend to develop mainly a cardiovascular (wet) beriberi, whereas Europeans tend to develop a dry beriberi with polyneuropathy and Wernicke's encephalopathy (22). In developed countries, most cases of Wernicke's encephalopathy are in alcoholics, although there is no clear correlation between the prevalence of Wernicke's encephalopathy and the per capita consumption of alcohol (23).

\subsection{Factors Affecting the Availability of Thiamine to the Brain in Alcohol Dependence}

\subsubsection{Genetic Predisposition}

Some individuals are probably genetically predisposed to developing WE. Alcoholics may have genetic defects in thiamine transport (24) which only becomes evident if they drink sufficient alcohol thereby limiting the availability of thiamine and causing problems with transport systems and storage. Two genes involved in the cellular transport of thiamine have recently been established: THTR1 (product SLC19A2) the high affinity transporter and THTR2 (product SLCA3) the low affinity transporter and we have identified changes in the genes of some WE patients which could potentially affect gene expression $(25,26)$. This functional impairment could contribute to an individual's ability to cope with thiamine deficiency or respond to therapy (27). Thus has important implications for the pharmacological treatment of Wernicke- Korsakoff Syndrome

\subsubsection{Inadequate Thiamine Intake}

Individuals with alcohol dependence typically neglect their diet and eat poorly. Often ingested nutrients are lost through vomiting, diarrhea or steatorrhea. The total body stores of thiamine are small, being approximately $30 \mathrm{mg}$ (daily requirements $1-2 \mathrm{mg}$, increased by alcohol metabolism) and will be depleted within a few weeks by poor intake even if absorption from the gastrointestinal tract remains normal.

\subsubsection{Impaired Absorption of Thiamine}

It was demonstrated in the 1960 s that alcohol can interfere with the absorption of thiamine in man which requires an active transport system. Further malabsorption occurs as malnutrition becomes a significant additional factor $(28,29$, 30). Alcohol-related liver damage reduces storage of thiamine, and thiamine phosphorylation is impaired. When malabsorption due to alcohol and malnutrition becomes a significant factor, this accelerates the decline, often rendering treatment with oral thiamine inadequate $(31,32)$

\subsubsection{Thiamine Transport Problems}

The damage caused by the combination of thiamine deficiency and alcohol metabolism probably interferes with adequate thiamine transport at a number of sites in the body, including the blood-brain barrier, as well as causing damage to the apo-enzymes which then require higher concentrations of thiamine to work normally (33). The accumulated damage means that treatment with oral thiamine is inadequate since it is unable to produce high enough concentrations of thiamine in the blood to traverse the blood-brain barrier (34). This is in marked contrast to the findings in Wernicke's Encephalopathy due to dietary deficiency alone, as these individuals do not seem to have significant problems with thiamine transport and therefore respond to small doses of thiamine.

\subsubsection{Other Nutritional Deficiencies}

In addition to the effects of ethanol on the intestinal transport of thiamine, other nutritional deficiencies found in chronic alcoholics may also impair absorption therefore all deficient nutrients should be replaced. Magnesium as a cofactor has a crucial role in the proper catalytic action of many enzymes, including transketolase in the pentose phosphate pathway (35) and thiamine pyrophosphokinase in the conversion of thiamine into thiamine pyrophosphate (35). Moreover, its severe deficiency may lead to a refractory response to thiamine until magnesium is given (36). 
Alcoholics can develop Wernicke's encephalopathy due to Magnesium deficiency.

Where WE is due to thiamine deficiency alone (i.e. in the absence of alcohol misuse) KP rarely supervenes following thiamine replacement therapy. Successful treatment or prophylaxis of WE in alcohol dependence probably depends on a number of inter-related issues and is not simply a matter of early and adequate thiamine treatment. If sufficient alcohol-related neurotoxicity has occurred by the time of diagnosis, then this may be the more important or limiting factor with respect to the long-term outcome. This possible obstacle to complete recovery should not prevent every attempt being made to provide the patient with optimum brain thiamine replacement (24).

\subsection{Biochemistry and Pathophysiology of Disease}

Thiamine requirement is directly related to both total caloric intake and the proportion of calories provided as carbohydrates (37). Thus, high caloric and high carbohydrate diets increase the demand for thiamine. The recommended dose of thiamine for an average, healthy adult is $1.4 \mathrm{mg}$ per day or $0.5 \mathrm{mg}$ of thiamine per $1000 \mathrm{kcal}$ consumed (I.C.M.R1990). This dose is higher in children, in ill conditions, and during pregnancy and lactation (38). In healthy individuals, total amount of thiamine that can be absorbed from a single oral dose is about $4.5 \mathrm{mg}$, as thiamine is absorbed in the duodenum by a rate-limited process (39). At the bloodbrain barrier, transport occurs by both passive and active mechanisms (39), which allows a rapid correction of brain thiamine deficiency, mainly by passive diffusion, if a steep concentration gradient between plasma and the brain is maintained, that can be achieved after parentral administration of the vitamin (39).

The main source of thiamine in diet of Indian population is cereals (rice and wheat) which contribute to $60-85 \%$ of the total supply. Thiamine is readily lost from rice during the process of milling. Being a water soluble vitamin, further losses take place during washing and cooking of rice. Much of thiamine in fruits and vegetables in generally lost during prolonged storage.

The body's stores of thiamine, usually depletes after 3 weeks of thiamine deficiency (40) and the blood levels of thiamine also fall (41) leading to impaired function of enzymes requiring thiamine pyrophosphate as a coenzyme $(40,42)$. In neuronal and glial cells, thiamine is converted to thiamine pyrophosphate, which is necessary for several biochemical pathways in the brain, such as intermediate carbohydrate metabolism (for energy production by ATP synthesis), lipid metabolism (for production and maintenance of myelin sheath), and production of amino acids and glucose-derived neurotransmitters (eg, glutamic acid; GABA)(43). Thiamine also seems to have a role in acetyl cholinergic and serotoninergic synaptic transmission and axonal conduction (44). Essential enzymes involved in the Krebs cycle and the pentose phosphate pathway are the $\alpha$ ketoglutarate-dehydrogenase, pyruvate dehydrogenase, and transketolase respectively (45). After about 4 days of thiamine deficiency, the $\alpha$-ketoglutarate-dehydrogenase activity is decreased in astrocytes leading $(46,47)$ to early damage to glial cells rather than neurons $(48,49)$. A reduction in the activity of transketolase is seen after about 1 week of thiamine deficiency, whereas no change in the activity of pyruvate dehydrogenase is observed for up to 10 days (46). Its metabolic impairment produces a diffuse decrease in the use of glucose in the brain with consequent severe impairment of cellular energy metabolism (50). In particular, many astrocyte-related functions are impaired, such as the control of intracellular and extracellular glutamate concentrations (with probable occurrence of glutamate-mediated excitotoxicity), the maintenance of ionic gradients across the cell membrane, and blood - brain barrier permeability (51).

Moreover, in Wernicke's encephalopathy at the symptomatic stage, increased lactate production by both neurons and astrocytes has been noticed, with intracellular accumulation of lactate, reductions in $\mathrm{pH}$, and focal acidosis (52). DNA fragmentation in thalamic neurons resulting in apoptotic cell death appears after about 2 weeks of thiamine deficiency (53). Other mechanisms involved include mitochondrial dysfunction and intracellular oxidative stress with production of free radicals and cytokines as a result of early endothelial-cell dysfunction and increased production of nitric oxide (54). The main consequence of these metabolic changes is the loss of osmotic gradients across cell membranes, with cytotoxic oedema and a progressive cellvolume increase firstly in astrocytes, then in neurons (55). Decreased $\alpha$-ketoglutarate-dehydrogenase activity resulting from thiamine deficiency, changes in the synthesis of amino acids, and the accumulation of lactate in the brain are initially reversible after prompt and sufficient thiamine therapy-the so-called stage of "reversible biochemical lesion" (51). Conversely, a lack or delay of thiamine rehabilitation may lead to structural, irreversible lesions in selective regions of the brain with possible permanent neurological sequelae or a fatal outcome (19).

Some studies report that neuroimaging findings can improve the early diagnosis of Wernicke's encephalopathy (WE) in clinical settings. The most distinctive neuroimaging finding of acute WE are cytotoxic edema and vasogenic edema, which are represented by bilateral symmetric hyperintensity alterations on T2-weighted MR images in the periphery of the third ventricle, periaqueductal area, mammillary bodies and midbrain tectal plate. An initial bout of WE can result in Korsakoff's syndrome (KS), but repeated bouts in conjunction with its typical comorbidity, chronic alcoholism, can result in signs of tissue degeneration in vulnerable brain regions. Chronic abnormalities identified with neuroimaging enable examination of brain damage in living patients with $\mathrm{KS}$ and have expanded the understanding of the neuropsychological deficits resulting from thiamine deficiency, alcohol neurotoxicity, and their co morbidity. Brain structure and functional studies indicate that the interactions involving the thalamus, mammillary bodies, hippocampus, frontal lobes, and cerebellum are crucial for 
memory formation and executive functions, and the interruption of these circuits by WE and chronic alcoholism can contribute substantially to the neuropsychological deficits in KS (56).

Repeated bouts of drinking and withdrawal are likely to lead to glutamate-induced excitability and lasting neuronal damage (57). The demand for thiamine is likely to be increased in the alcoholic brain because of increased requirements for alcohol metabolism, which may be further increased during alcohol withdrawal. Thiamine deficiency also causes excessive glutamate release so when it occurs in combination with alcohol- induced glutamate receptor up regulation, the potential for neurotoxicity is increased. It is no accident that case reports describing episodes of WE show that it typically occurs during a period of sudden unexpected and untreated alcohol withdrawal. A number of authors have suggested that thiamine deficiency may interact synergistically with alcohol neurotoxicity to promote progression from WE to Korsakoff Syndrome (19, 58, 59). Korsakoff Syndrome (KS) is unlikely to be caused purely by thiamine deficiency. Failure to respond to thiamine may in part be due to the fact that cells have been irreversibly damaged by lack of thiamine, but could also be due to deficiencies of other nutrients and alcohol-induced neurotoxicity.

\subsection{Clinical Presentation}

The classic clinical triad of Wernicke's encephalopathy consists of mental status changes, ophthalmoplegia, and gait ataxia. However, the complete triad may be present in as few as $10 \%$ of cases (13). Conversely, other signs of disease such as hypothermia, vestibular dysfunction, and other ocular abnormalities can be present $(19,37,60)$. In fact, nystagmus is the most common ocular abnormality, not complete ophthalmoplegia $(19,39)$. Thus, reliance on the presence of the clinical triad as the sole criterion for disease is often inadequate and may lead to under diagnosis. The most constant component of disease when determined from retrospective autopsy studies is mental status changes (14, 21). The degree and nature of cognitive impairment range from apathy or mild confusion to complete coma (19). Although coma is a rare presentation, comatose patients are often critically ill and perhaps more likely to go unrecognized $(39,40,41,61)$. The second component of the classic triad was originally reported to be ophthalmoplegia; however, ophthalmoplegia remains a whole range of ocular findings that include nystagmus, sixth nerve palsy, ptosis, retinal hemorrhage, papilledema, and (less commonly) anisocoria or miosis $(19,38)$. Complete ophthalmoplegia rarely occurs and likely represents progressive disease, and nystagmus (usually horizontal) is the most common ocular abnormality (19). Thus, for a more accurate and inclusive description of disease, the term "ocular" should replace ophthalmoplegia in the clinical triad. The final component of the triad is gait ataxia, and this finding may range from mild gait disturbance to a complete inability to stand. Several other findings may occur. Patients may be critically ill with hypotension, hypothermia, and obtundation (62, 63, 64). Lactic acidosis, oftentimes severe, may occur with or without Wernicke's encephalopathy in thiamine-deficient states. Sudden death, possibly associated with exertion, has occurred rarely (65). Other associated clinical findings of thiamine-deficient states may include cardiac failure (typically high output), gastrointestinal complaints (abdominal pain, nausea, vomiting), and polyneuropathy. Deafness has also been reported in Wernicke's encephalopathy, as well as vestibular dysfunction noted by the subtle finding of an abnormal response to caloric testing.

\subsection{Clinical Features of Wernicke's Encephalopathy}

Common symptoms or signs at presentation [ (66),Adapted from Thomson et al. 2009]

- Ocular abnormalities

- Mental status changes

- In-coordination of gait and trunk ataxia

Uncommon symptoms or signs at presentation

- Stupor

- Hypotension and tachycardia

- Hypothermia

- Bilateral visual disturbances and papilloedema

- Epileptic seizures

- Hearing loss

- Hallucinations and behavioural disturbances

Late-stage symptoms

- Hyperthermia

- Increased muscular tone and spastic paresis

- Choreic dyskinesias

- Coma

Operational criteria for Wernicke's Encephalopathy (67): 2 of the following

- Dietary deficiencies

- Oculomotor abnormalities

- Cerebellar dysfunction

- Either altered mental state or mild memory impairment

\subsection{Diagnosis}

Prompt diagnosis of this syndrome is vital because if undiagnosed or inadequately treated, it is likely to proceed to Korsakoff syndrome. Therefore, the initial diagnosis of Wernicke's encephalopathy remains clinical. A recent operational definition derived from a retrospective study with a cohort of known alcoholics stated that any 2 of the following 4 conditions should be sufficient for a presumptive diagnosis: nutritional deficiency, ocular findings, ataxia, and mental status changes (67). This operational definition may help guide physicians and is intended to increase diagnostic yield, but the full range of potential clinical presentation should always be considered, including hypotension, hypothermia, and coma. Over diagnosis rather than under diagnosis is preferred because the consequences of misdiagnosis are grave. Thiamine levels can be measured either directly or indirectly but take several days to perform and will not aid indecision making in the emergency 
department. However, the thiamine concentration could help the clinician assuming care of the patient in ambiguous cases, and obtaining a thiamine level can be considered before administration of the vitamin. Diagnosis of Wernicke's encephalopathy is not contingent on direct or indirect thiamine levels, because the sensitivity or specificity of a thiamine level for active disease has not been determined. An MRI offer the best way to make a definitive diagnosis ante mortem, but the sensitivity is poor and obtaining an MRI for this indication is typically impractical and unnecessary in the emergency department . The reported sensitivity of MRI was $53 \%$ and specificity $93 \%$ for active disease, but the results were not compared to postmortem diagnosis and the sample size was small. The same study reported $13 \%$ sensitivity for computed tomography scanning (68).

\subsection{Differential Diagnosis}

Several pitfalls exist in differentiating Wernicke's encephalopathy from other disease processes. Firstly, iatrogenic exacerbation of Wernicke's encephalopathy can occur with prolonged glucose or carbohydrate loading in the absence of adequate thiamine. A single acute administration of glucose does not appear to cause this effect $(3,69,70$, 71,72). Thus, nutritionally deficient patients receiving glucose should also receive thiamine, but urgent administration of glucose should not be with held pending thiamine administration. Secondly, many medical conditions can imitate Wernicke's encephalopathy, and these should not be overlooked, particularly if the patient has no clear history of nutritional deficiency and the MRI signal characteristics and lesion sites are not entirely specific for Wernicke's encephalopathy (73). Therefore, other possible causes of acute encephalopathies need to be differentiated, particularly when the clinical history does not reveal a definite predisposing factor related to Wernicke's encephalopathy, or when the response of neurological signs to the administration of thiamine is unclear $(74,75,76)$. Third, Wernicke's encephalopathy can develop in predisposed populations because of increased metabolic demand caused by other disease states, analogous to an underlying disease precipitating the development of diabetic ketoacidosis. The most common inciting factor contributing to Wernicke's encephalopathy is infection. Thus, concomitant diseases such as pneumonia or even meningitis do not exclude a diagnosis of Wernicke's encephalopathy (19).Conversely, meningitis or encephalitis may mimic Wernicke's encephalopathy. Alcoholic are particularly predisposed to Wernicke's encephalopathy because of inadequate dietary intake, reduced gastrointestinal absorption, and decreased hepatic storage.

\subsection{Management}

Wernicke's encephalopathy is a medical emergency, and in patients in whom the disorder is suspected thiamine should be initiated immediately, either intravenously or intramuscularly, to ensure adequate absorption (44). Because thiamine hydrochloride can be inactivated by heat, its solutions should be fresh. It is mandatory that thiamine is given before or concomitantly with intravenous administration of glucose when a diagnosis of Wernicke's encephalopathy is suspected, because glucose alone can precipitate the disorder in thiamine-deficient individuals. This is an absolute necessity for patients who have been drinking alcohol and now present with hypoglycemia (39). Moreover, to prevent the development of Korsakoff syndrome and to treat symptoms of Wernicke's encephalopathy, in all patients with any evidence of chronic alcohol misuse and a poor diet, parenteral thiamine treatment should be initiated immediately while the patients are still drunk and continued for an adequate time for any patient with evidence of Wernicke's encephalopathy when sober (78) Victor and co-workers (20) found that recovery from the ophthalmoplegia was complete after a few hours, except for a residual, fine, horizontal nystagmus in $60 \%$ of patients. Recovery from ataxia occurred after a few days, although in some cases was incomplete. The changes in mental status tended to improve after $2-3$ weeks of therapy. Deficiency in other vitamins and electrolytes, especially niacin and magnesium, should also be corrected. The brain requires a continuous supply of thiamine for metabolic and synthetic purposes. At normal plasma concentrations of thiamine, influx into the brain is almost entirely due to a high affinity carrier mediated process. The uptake occurs at $0.3 \mathrm{ug} / \mathrm{h} / \mathrm{g}$ tissue (77) and is very similar to the rate of thiamine turnover in the brain (0.3-0.5 microgram $/ \mathrm{h} / \mathrm{g}$ tissue) (78). It is just sufficient to meet the brain's requirements, making the brain very vulnerable to any fall in circulating levels of thiamine. Passive diffusion can occur but requires high concentrations of thiamine probably not achieved by oral therapy in a malnourished alcoholic patient (79). The requirement for thiamine will be increased if damage has occurred to the apoenzymes and may be a contributory factor to the development of WE and KS $(80,81)$. It is also possible that so-called "normal" circulating levels of thiamine may not be adequate if the transport system is genetically abnormal and the enzyme systems within the neuron are damaged. That may explain, at least in part, why alcoholic patients with WE require such large parenteral doses of thiamine.

Treatment recommendations for WE have been determined from evidences derived from case reports and reviews (82). Doses of parenteral thiamine between $100 \mathrm{mg}-250 \mathrm{mg}$ do not always prevent death, and between $56 \%-84 \%$ of patients with WE are found to develop KS if followed up long-term $(17,83)$. This poor outcome is not necessarily due to irreversible brain damage having been present at the time of presentation. Other studies show that these doses are suboptimal and may not restore vitamin status, or improve clinical signs or prevent death (21).

Cochrane review indicates that there is insufficient evidence from randomized controlled trials to guide the clinician as to the optimum dose, frequency, route, or duration of thiamine treatment for prophylaxis or treatment of Wernicke's encephalopathy due to alcohol misuse. 
However, the doses of thiamine as mentioned below, are recommended by the British National Formulary and the Royal College of Physicians, London (Royal College of Physicians 2001) and have been licensed for use in the UK by the Medicines and Healthcare Products Regulatory Agency (MHRA) since 1994. They are also in accordance with the evidence-based guidelines published by the British Association for Psychopharmacology (84), EFNS (85), NICE guidelines (86).

- Thiamine $500 \mathrm{mg}$ IV three times daily for 2-to-3 days and $250 \mathrm{mg}$ IV daily for the next 3-to-5 days given as an infusion over $30 \mathrm{~min}$, diluted in $50-100 \mathrm{~mL}$ of normal saline

- Thiamine $100 \mathrm{mg}$ orally three times daily for the rest of the hospital stay and during outpatient treatment. Absorption will be $<4.5 \mathrm{mg}$ daily

- Multivitamins IV

- Replace magnesium: average deficit $2 \mathrm{mEq} / \mathrm{kg}$

- Replace fluid and electrolyte losses: monitor electrolytes, blood pressure and renal function

Prophylactic treatment - intramuscular administration of $250 \mathrm{mg}$ thiamine once daily for $3-5$ consecutive daysshould be used in all people with severe alcohol withdrawal, poorly nourished patients, and people with poor diet and signs of malnutrition $(39,87,88,89)$. Because a higher enteral intake of thiamine is non toxic, thiamine supplementation by mouth should be continued for several months at a dose of 30 mg twice daily.

\subsection{Parenteral Thiamine and Unwanted Side-Effects}

Parenteral thiamine administration is generally safe. In a prospective assessment of the safety of thiamine hydrochloride, given as a $100 \mathrm{mg}$ intravenous bolus in 989 consecutive patients (1070 doses), Wrenn and colleagues (90) found one patient with generalized pruritis (major reaction, $0.093 \%$ ) and 11 with transient local irritation (minor reactions, $1.02 \%)(94)$. Moreover, in a retrospective study Wrenn and Slovis (91) found no significant allergic reactions in more than 300000 patients treated with parenteral thiamine. However, anaphylactic reactions (i.e., a true allergic reaction to thiamine) or anaphylactic reactions (i.e., a dose-dependent reaction) may occur occasionally when thiamine is given parenterally (39). In particular, serious allergic adverse reactions may occur during or shortly after parenteral administration, mainly with intravenous administration. These include anaphylactic shock (rarely fatal), dyspnoea and bronchospasm and cutaneous rash or flushing $(39,87)$. Because the unwanted side-effects to B vitamins are most commonly seen after multiple administrations when given intravenously, rather than a slow infusion $(92,93)$. Thiamine should be given intravenously diluted with $100 \mathrm{~mL}$ of normal saline or $5 \%$ glucose, and infused over $30 \mathrm{~min}(39,87)$. Facilities for treating anaphylaxis should be available when thiamine is given, including facilities for cardiopulmonary resuscitation and immediately available intravenous (1:10 000) or intramuscular $(1: 1000)$ adrenaline $(39,87)$.

\subsection{Prognosis}

Administration of thiamine improves the conditions however, persistent neurologic dysfunction is common and the reported mortality is $17 \%$ (21). Ophthalmoplegia typically resolves, rapidly. The initial state of global confusion usually improves within hours or days. Studies in alcoholics have been reported that $80 \%$ or more of Wernicke's encephalopathy patients may progress to a Korsakoff state (19). A recent report by Chiossi et al. (7) of Wernicke's encephalopathy patients with hyperemesis gravidarum revealed that many patients also had cognitive and memory impairment . Complete resolution occurred only in only 14 of 49 cases. Similar persistent neurologic deficits have been found in the alcoholic population, although other factors such as increased rates of trauma, hypoglycemia, and comorbid disease may play a role in alcoholic patients (19).Main residual findings from Wernicke's encephalopathyare nystagmus, gait ataxia, and the Korsakoff syndrome.

\section{Conclusion}

Wernicke's encephalopathy (WE) is an acute neuropsychiatric condition caused by an inadequate supply of thiamine (vitamin B1) to the brain which is associated with significant morbidity and mortality. In developing country, there are more chances of thiamine deficiency, because of poor intake of nutrients in routine diet due to economic reasons and also local costumes and cultural practices concerning the processing and cooking of rice and other foodstuffs. The national household survey of drug use in India (2004) about prevalence of alcohol use also shows that about $21.4 \%$ of male between $18-40$ age group consuming alcohol and Individuals with alcohol dependence typically neglect their diet and eat poorly . Most our emergency physicians and general practitioners are not well sensitized about neuropsychiatric disorder in alcoholic brain. Till date no such studies are available about treatment of WernickeKorsakoff syndrome in Indian population. Therefore all these added factors make them more vulnerable to thiamine deficiency leading to Wernicke-Korsakoff syndrome. Unfortunately the disorder is still greatly under diagnosed in both adults and children because of either a relatively nonspecific clinical presentation of the disease in some cases, or because of unrecognized clinical data and neurological signs. The diagnosis is clinical and is mainly supported by the dramatic response of neurological signs to parenteral thiamine. In recent studies MRI is currently considered the most valuable for diagnosis.

Although alcoholism is the most common predisposing factor but Wernicke's encephalopathy can occur in any patient with nutritional deficiency conditions such as hyperemesis gravidarum, hemodialysis, malignancy, use of total parenteral nutrition without adequate thiamine, and abdominal surgery. To educate clinicians, specially emergency physicians about evaluation and treatment of 
Wernicke-Korsakoff syndrome is as important as to educate people to eat well balanced, mixed diet containing thiamine rich food (e.g., parboiled, under milled rice and wheat whole), because most of these patients visit to emergency department and are frequently unrecognized . Further clinician's knowledge of this syndrome is vital because if undiagnosed or inadequately treated, it is likely to proceed to Korsakoff syndrome. Therefore, best treatment for WernickeKorsakoff syndrome is prompt diagnosis and adequate treatment.

Further studies are required to define the mechanisms of neuronal cell death in Wernicke's encephalopathy and also, randomized controlled trials are needed to define the optimum dose and duration of thiamine treatment for prophylaxis or treatment of Wernicke's encephalopathy and Korsakoff syndrome in people with chronic alcoholism and in people without alcoholism. Future research in the identification of patients who may be genetically predisposed to Wernicke's encephalopathy, so that prophylaxis may be given to this population to prevent Wernicke's encephalopathy and its complications.

\section{References}

[1] Campbell ACP, Russel WR. Wernicke's encephalopathy: the clinical features and their probable relationship to vitamin B deficiency. Q J Med 1941; 10: 41-64.

[2] Ihara M, Ito T, Yanagihara C, et al. Wernicke's encephalopathy associated with hemodialysis: report of two cases and review of the literature. Clin Neurol Neurosurg. 1999; 101:118-121.

[3] Wagener H, Weir J. Ocular lesions associated with postoperative and gestational nutritional deficiency. Am J Ophthalmol. 1937; 20:253-59.

[4] Toth C, Voll C. Wernicke's encephalopathy following gastroplasty for morbid obesity. Can J Neurol Sci. 2001; 28:89-92.

[5] Michowitz Y, Copel L, Shiloach E, et al. Non-alcoholic Wernicke's encephalopathy: unusual clinical findings. Eur J Intern Med.2005; 16:443-44.

[6] Munir A, Hussain SA, Sondhi D, et al. Wernicke's encephalopathy in a non-alcoholic man: case report and brief review. Mt SinaiJ Med. 2001; 68:216-18.

[7] Chiossi G, Neri I, Cavazzuti M, et al. Hyperemesis gravidarum complicated by Wernicke encephalopathy: background, case report, and review of the literature. Obstet Gynecol Surv. 2006; 61:255-68.

[8] Harper C. The incidence of Wernicke's encephalopathy in Australia - a neuropathological study of 131 cases. J Neurol Neurosurg Psychiatry. 1983; 46:593-98.

[9] Harper C. Wernicke's encephalopathy: a more common disease than realised. A neuropathological study of 51 cases. J Neurol Neurosurg Psychiatry. 1979; 42:226-31.

[10] Bleggi-Torres LF, de Medeiros BC, Werner B, et al. Neuropathological findings after bone marrow transplantation: anautopsy study of 180 cases. Bone Marrow Transplant. 2000; 25:301-07.

[11] Torvik A. Wernicke's encephalopathy-prevalence and clinical spectrum. Alcohol Alcohol Suppl. 1991; 1:381-84.

[12] Boldorini R, Vago L, Lechi A, et al. Wernicke's encephalopathy:occurrence and pathological aspects in a series of 400 AIDS patients. Acta Biomed Ateneo Parmense. $1992 ; 63: 43-49$

[13] Harper CG, Giles M, Finlay-Jones R. Clinical signs in the Wernicke-Korsakoff complex: a retrospective analysis of 131 cases diagnosed at necropsy. J Neurol Neurosurg Psychiatry 1986; 49: 341-45.

[14] Lana-Peixoto MA, Dos Santos EC, Pittella JE. Coma and death in unrecognized Wernicke's encephalopathy. An autopsy study. Arq Neuropsiquiatr. 1992; 50:329-33.

[15] Vasconcelos MM, Silva KP, Vidal G, et al. Early diagnosis of pediatric Wernicke's encephalopathy. Pediatr Neurol 1999; 20: 289-94.

[16] Torvik A, Lindboe CF, Rogde S. Brain lesions in alcoholics. A neuropathological study with clinical correlations. J Neurol Sci.1982; 56:233-48.

[17] Harper CG, Sheedy DL, Lara AI, et al. Prevalence of Wernicke-Korsakoff syndrome in Australia: has thiamine fortification made a difference? Med J Aust. 1998; 168:542-45.

[18] Naidoo DP, Bramdev A, Cooper K. Autopsy prevalence of Wernicke's encephalopathy in alcohol-related disease. S Afr Med J. 1996; 86:1110-12.

[19] Victor M, Adams RD, Collins GH. The Wernicke-Korsakoff Syndrome and Related Neurologic Disorders Due to Alcoholism and Malnutrition. 2nd ed. Philadelphia, PA: FA Davis; 1989.

[20] Victor M. The Wernicke-Korsakoff syndrome. In: Vinken PJ, Bruyn GW, eds. Handbook of clinical neurology, vol 28, part II. Amsterdam: North-Holland Publishing Company, 1976: 243-70.

[21] Victor M, Adams RD, Collins GH. The Wernicke-Korsakoff syndrome: a clinical and pathological study of 245 patients, 82 with post-mortem examinations. Contemp Neurol Ser 1971; 7 : 1-206.

[22] Blass JP, Gibson GE. Abnormality of a thiamine requiring enzyme in patients with Wernicke-Korsakoff Syndrome. N Engl J Med 1977; 297: 1367-70.

[23] Harper C, Fornes P, Duyckaerts C, et al. An international perspective on the prevalence of the Wernicke-Korsakoff syndrome. Metab Brain Dis 1995; 10: 17-24.

[24] Thomson AD, Marshall EJ. The natural history and pathophysiology of Wernicke's encephalopathy and Korsakoff's psychosis. Alcohol Alcohol 2006; 41: 151-58.

[25] Guerrini I, Thomson AD, Cook CC, et al. Direct genomic PCR sequencing of the high affinity thiamine transporter (SLC19A2) gene identifies three genetic variants in Wernicke Korsakoff syndrome (WKS) AmJ Med Genet B Neuropsychiatr Genet. 2005; 137:17-19.

[26] Guerrini I, Thomson AD, Gurling HM. Molecular genetics of alcohol related brain damage. Alcohol and Alcoholism. 2009; 44:166-70. 
[27] Singleton CK, Martin PR. Molecular mechanisms of thiamine utilization. Curr Mol Med 2001; 1: 197-207.

[28] Thomson AD. The absorption of sulphur-labelled thiamine hydrochloride in control subjects and in patients with intestinal malabsorption. Clinical Science 1966; 31(2): 167-79.

[29] Thomson AD, Baker H, Leevy CM. Patterns of 35S-thiamine hydrochloride absorption in the malnourished alcoholic. Journal of Laboratory and Clinical Medicine 1970; 76(1): 34 45.

[30] Thomson $A D$, Jeyasingham $M$, Pratt $\mathrm{OE}$, et al. Nutrition and alcoholic encephalopathies. ActaMedicaScandinavica. Supplementum. 1987; 717: 55-65.

[31] Thomson AD. Mechanisms of vitamin deficiency in chronic alcohol misusers and the development of the WernickeKorsakoff Syndrome. Alcohol and Alcoholism. 2000; 35(1):27.

[32] Thomson AD, Marshall EJ. The treatment of patients at risk of developing Wernicke's encephalopathy in the community. Alcohol and Alcoholism. 2006; 41:159-67.

[33] Harper C, Dixon G, Sheedy D, et al. Neuropathological alterations in alcoholic brains. Studies arising from the New South Wales Tissue Resource Centre. Prog Neuropsychopharmacol Biol Psychiatry. 2003; 27:951-61.

[34] Thomson AD, Cook CCH, Touquet R, et al. The Royal College of Physicians report on alcohol: guidelines for managing Wernicke's encephalopathy in the Accident and Emergency Department. Alcohol and Alcoholism. 2002;37:513-21.

[35] Zieve I. Influence of magnesium deficiency on the utilization of thiamine. Ann NY Acad Sci 1969; 162: 732-43.

[36] Traviesa DC. Magnesium deficiency: a possible cause of thiamine refractoriness in Wernicke-Korsakoff encephalopathy. J Neurol Neurosurg Psychiatry 1974; 37: 959-62.

[37] Sauberlich HE, Herman YF, Stevens CO, Herman RH. Thiamine requirement of the adult human. Am J Clin Nutr 1979; 32: 2237-48.

[38] Davis RE, Icke GC. Clinical chemistry of thiamine. Adv Clin Chem 1983; 17: 93-140.

[39] Thomson AD, Cook CCH, Touquet R,et al. The Royal College of Physicians report on alcohol: guidelines for managing Wernicke's encephalopathy in the accident and emergency department. Alcohol Alcohol Suppl 2002; 37: 513-21.

[40] Tanphaichitr V. Thiamin. In: Shils ME, Olson JA, Shike M, Ross AC, eds. Modern Nutrition in Health and Disease. Baltimore MD: Williams and Wilkins, 1999: 381-89.

[41] Leevy CM. Thiamin deficiency and alcoholism. Ann N Y Acad Sci 1982; 378: 316-26.

[42] Schenker S, Henderson GI, Hoyumpa AM, et al. Hepatic and Wernicke's encephalopathies: current concepts of pathogenesis. Am J Clin Nutr 1980; 33: 2719-26.

[43] Manzo L, Locatelli C, Candura SM, et al. Nutrition and alcohol neurotoxicity. Neurotoxicology 1994; 15: 555-65.

[44] Iwata H. Possible role of thiamine in the nervous system. Trends Pharmacol Sci 1982; 4: 171-73.

[45] McCandless DW, Schenker S, Cook M. Encephalopathy of thiamine deficiency: studies of intracerebral mechanisms. J Clin Invest 1968; 47: 2268-80.

[46] Butterworth RF. Cerebral thiamine-dependent enzyme changes in experimental Wernicke's encephalopathy. Metab Brain Dis 1986; 1: 165-75.

[47] Hazell AS, Pannunzio P, Rama Rao KV, et al. Thiamine deficiency results in downregulation of the GLAST glutamate transporter in cultured astrocytes. Glia 2003; 43: 175-84.

[48] Collins GH. Glial cell changes in the brain stem of thiaminedeficient rats. Am J Pathol 1967; 50: 791- 814.

[49] Robertson DM, Wasan SM, Skinner DB. Ultrastructural features of early brain stem lesions of thiamine-deficient rats. Am J Pathol 1968; 52: 1081-97.

[50] Hakim AM, Pappius HM. Sequence of metabolic, clinical, and histological events in experimental thiamine deficiency. Ann Neurol 1983; 13: 365-75.

[51] Hazell AS, Todd KG, Butterworth RF. Mechanisms of neuronal cell death in Wernicke's encephalopathy. Metab Brain Dis 1998; 13: 97-122.

[52] Navarro D, Zwingmann C, Hazell AS, et al. Brain lactate synthesis in thiamine deficiency: a re-evaluation using $1 \mathrm{H}-$ 13C nuclear magnetic spectroscopy. J Neurosci Res 2005; 79: $33-41$.

[53] Matsushima K, MacManus JP, Hakim AM. Apoptosis is restricted to the thalamus in thiamine-deficient rats. Neuroreport 1997; 8: 867-70.

[54] Desjardins P, Butterworth RF. Role of mitochondrial dysfunction and oxidative stress in the pathogenesis of selective neuronal loss in Wernicke's encephalopathy. Mol Neurobiol 2005; 31: 17-25.

[55] Chan F, Butterworth RF, Hazell AS. Primary cultures of rat astrocytes respond to thiamine deficiency-induced swelling by downregulating aquaporin-4 levels. Neurosci Lett 2004; 366: 231-34.

[56] Jung YC, Chanraud S, Sullivan EV. Neuroimaging of Wernicke's encephalopathy and Korsakoff's syndrome. Neuropsychol Rev. 2012; 22(2):170-80.

[57] Brust JCM. Ethanol and cognition: indirect effects, neurotoxicity and neuroprotection: a review. International Journal of Environmental Research Public Health. 2010; $7: 1540-57$.

[58] Wood B. Thiamin status in Australia. World Review of Nutrition and Dietetics. 1985; 46:148-218.

[59] Victor M, Adams RD (1985). The alcoholic dementias. In P. J. Vinken, G. W. Bruyn, \& H. L. Klawans (Eds.), Handbook of clinical neurology (Vol 46, pp. 335-353). Amsterdam: Elsevier.

[60] Reuler JB, Girard DE, Cooney TG. Current concepts. Wernicke's encephalopathy. N Engl J Med. 1985; 312:103539.

[61] De Keyser J, Deleu D, Solheid C, et al. Coma as presenting manifestation of Wernicke's encephalopathy. J Emerg Med.1985; 3:361-63.

[62] Kearsley JH, Musso AF. Hypothermia and coma in the Wernicke-Korsakoff syndrome. Med J Aust. 1980; 2:504-06. 
[63] Donnan GA, Seeman E. Coma and hypothermia in Wernicke's encephalopathy. Aust N Z J Med. 1980; 10:438-39.

[64] Ackerman WJ. Stupor, bradycardia, hypotension and hypothermia. A presentation of Wernicke's encephalopathy with rapid response. West J Med 1974; 121:428-9.

[65] Fried RT, Levy M, Leibowitz AB, et al. Wernicke's encephalopathy in the intensive care patient. Crit Care Med. 1990; 18:779-80.

[66] Thomson AD, Guerrini I, Marshall encephalopathy: role of
Gastroenterology. 2009; 33(6):21-30.

JE. Wernicke's

[67] Caine D, Halliday GM, Kril JJ, et al. Operational criteria for the classification of chronic alcoholics: identification of Wernicke's encephalopathy. J Neurol Neurosurg Psychiatry. 1997; 62:51-60.

[68] Antunez E, Estruch R, Cardenal C, et al. Usefulness of CT andMR imaging in the diagnosis of acute Wernicke's encephalopathy. AJR Am J Roentgenol. 1998; 171:1131-37.

[69] Hack JB, Hoffman RS. Thiamine before glucose to prevent Wernicke encephalopathy: examining the conventional wisdom. JAMA. 1998; 279:583-84.

[70] Watson AJ, Walker JF, Tomkin GH, et al. Acute Wernicke's encephalopathy precipitated by glucose loading. Ir J Med Sci.1981; 150:301-03.

[71] Zimitat C, Nixon PF. Glucose loading precipitates acute encephalopathy in thiamin-deficient rats. Metab Brain Dis. 1999; 14:1-20.

[72] Zimitat C, Nixon PF. Glucose induced IEG expression in the thiamin-deficient rat brain. Brain Res. 2001; 892:218-27.

[73] Weidauer S, Nichtweiss M, Lanfermann H, et al. Wernicke's encephalopathy: MR findings and clinical presentation. Eur Radiol 2003; 13: 1001-09.

[74] Brechtelsbauer DL, Urbach H, Sommer $\mathrm{T}$, et al. Cytomegalovirus encephalitis and primary cerebral lymphoma mimicking Wernicke's encephalopathy. Neuroradiology 1997; 39: 19-22.

[75] Zurkirchen MA, Misteli M, Conen D. Reversible neurological complications in chronic alcohol abuse with hypophosphatemia. Scweiz Med Wochenschr 1994; 124: 1807-12.

[76] Squier MV, Thompson J, Rajgopalan B. Case report: neuropathology of methyl bromate intoxication. Neuropathol Appl Neurobiol 1992; 6: 579-84.

[77] Greenwood J, Love ER, Pratt OE. Carrier mediated transport of thiamine across the blood-brain Barrier. Proceedings of the Physiological Society, Journal of Physiology. 1980; 310:23.

[78] Rindi G, Patrini C, Comincioli V, et al. Thiamine content and turnover rates of some rat nervous regions, using labeled thiamine as a tracer. Brain Research. 1980; 81:369-80.

[79] Rolland S, Truswell AS. Wernicke-Korsakoff syndrome in Sydney hospitals after 6 years of thiamine enrichment of bread. Public Health Nutr 1998; 1: 117-22.

[80] Pratt OE, Jeyasingham M, Shaw GK, et al. Transketolase variant enzymes and brain damage. Alcohol and Alcoholism. $1985 ; 20: 223-32$.

[81] Heap LC, Pratt OE, Ward RJ, et al. Individual susceptibility to Wernicke-Korsakoff syndrome and alcoholism induced cognitive deficit: impaired thiamine utilization found in alcoholics and alcohol abusers. Psychiatric Genetics. 2002; $12: 217-24$.

[82] Nakada T, Knight RT. Alcohol and the central nervous system. The Medical Clinics of North America. 1984; 68:121-31.

[83] Wood B, Currie J, Breen K. Wernicke's encephalopathy in a metropolitan hospital: a prospective study of the incidence, characteristics and outcome. Medical Journal of Australia 1986; 144: 12-16.

[84] Lingford-Hughes AR, Welch S, Nutt DJ. Evidence based guidelines for the pharmacological management of substance misuse, addiction and comorbidity: recommendations from the British Association for Psychopharmacology. J Psychopharmacology. 2004; 18:293-335.

[85] Galvin R, Brathen G, Ivashynka A, et al. EFNS guidelines for diagnosis, therapy and prevention of Wernicke Encephalopathy. European Journal of Neurology. 2010; 17:1408-18.

[86] NICE (2011). Alcohol-use disorders: diagnosis, assessment and management of harmful drinking and alcohol dependence. Clinical Guideline 115. London: NICE.

[87] Cook CCH, Hallwood PM, Thomson AD. B-vitamin deficiency and neuro-psychiatric syndromes in alcohol misuse. Alcohol Alcohol Suppl 1998; 33: 317-36.

[88] Cook CC. Prevention and treatment of Wernicke-Korsakoff Syndrome. Alcohol Alcohol Suppl 2000; 35: 19-20.

[89] Bligh JG, Madden JS. Managing alcohol dependence. Practitioner 1983; 227: 82-85.

[90] Wrenn KD, Murphy F, zidovudine Slovis CM. A toxicity study of parenteral thiamine hydrochloride. Ann Emerg Med 1989; 18: 867-70.

[91] Wrenn KD, Slovis CM. Is intravenous thiamine safe?. Am J Emerg Med 1992; 10: 165.

[92] Leitner ZA. Untoward effects of vitamin B. Lancet 1943; 2: 474-75.

[93] Stein W, Morgenstern M. Sensitization to thiamine hydrochloride: report of a case. Ann Int Med 1994; 70: 826-28. 